\title{
Performance Evaluation of Voice and video conferencing For WIMAX Network under Various Modulation techniques
}

\author{
Almutaz Ali Mohammed ${ }^{1}$, Ibrahim Elimam Abdalla ${ }^{2}$ \\ ${ }^{1,2}$ Faculty of Engineering, Neelain University, Khartoum -Sudan
}

\begin{abstract}
Worldwide interoperability for Microwave Access (WIMAX) that based on 802.16d/e Orthogonal Frequency Division Multiplexing (OFDM) and based on adaptive Physical Layer (PHY), It provides wireless broadband to fixed and mobile terminals, The basic WIMAX feature is wireless transmission infrastructure which allows a fast use as well as low maintenance costs, Modulation techniques enable WIMAX network to be optimized. WIMAX supports BPSK, 16-QAM, DPSK, and 64-QAM as a modulation technique. This paper present evaluation of various modulation techniques and study of performance for voice and video conferencing applications, An Opnet software used to simulate WIMAX Modulation techniques impact on voice and video conferencing, The simulation results includes the performance analysis for bit error rate (BER), SNR, MOS, MOS loss Rate, end to end packet delay and Jitter of different modulation.
\end{abstract}

Keywords: WIMAX, BER, SNR, MOS loss Rate, Modulation, OFDM.

\section{Introduction}

Worldwide Interoperability for Microwave Access (WIMAX) is broadband wireless data communications technology based on IEEE 802.16 standard providing high-speed data. It is a new wireless OFDM (Orthogonal Frequency Division Multiplexing) technology that provides high quality broadband services, long distances spreading based on IEEE.802.16 without the selective fading and other issues of other forms of signal format [9],[3]. WIMAX Technology do a various types of task such as providing high-speed internet, telephone service, video streaming and voice application etc.

WIMAX uses a special type of modulation technique which is a blend of ASK and PSK with a new name called QAM (phase and amplitude changes at the same time). In OFDM smaller data stream is then mapped to sub-carrier data and modulated used sort of PSK or QAM such as QPSK, 16-QAM, 64-QAM. OFDM needs less bandwidth than Frequency Division Multiplexing (FDM). When compare throughput of WIMAX with $3 \mathrm{G}$, unlike $3 \mathrm{G}$ which have fixed channel, WIMAX depend on used channel bandwidth, the most licensed deployment spectrum of WIMAX either $2.5 \mathrm{G}$ or $3.5 \mathrm{G}$. WIMAX designed for flexibility and different sizes supported, as example $3.5 \mathrm{MHz}$ for fixed WIMAX and from $1.25 \mathrm{MHz}$ to $20 \mathrm{MHz}$ for mobile WIMAX. SNIR is reflecting the rate of information transfer rate [5]. The idea behind this paper is to study the usage of variety modulation with fix coding rate (1/2) per user when voice and video conferencing apps taken place. As part of WIMAX stander Adaptive modulation provide the system capability that make the best available channel conditions, The main idea from the Adaptive modulation is to dynamically adjust and adapt the transmission parameters, such as modulation and coding schemes, the physical layer of WIMAX(PHY) [2] described as the layer that can :-

Generate information of data rate, Scrambling, Forward error correction, Interleaving, Modulation, perform reverse operations to decode the received sequences of information bits and calculate the Bit Error Rate (BER) as function of against different values of signal to noise ratio (SNR) [1].In the rest of the paper, Section 2 briefly introduces the definition and features of TCP - UDP, voice call and videoconferencing (VC) applications. Section 3 describes the simulation model and the common parameters used in this study. While Section 4 describes various performance metrics used for evaluating the performance. Section 5 presents the simulation results and discussions, finally Section 6 show the conclusion of this study.

\section{Tcp /Udp- (Voice/Vc)}

TCP is a connection oriented and designed as reliable protocol that guarantees all data delivered in sequence and without loss, TCP is able to transfer a continuous stream in each direction between connected ends, duplication or delivering out-of-order is mechanism of recovering from data that damaged [10]. UDP is defined as connection-less to make available datagram mode of packet-switched computer communication Primarily for establishing, low-latency and loss tolerating connections between applications. Deep difference aspects and description for TCP/UDP in [4]. As audio and video conferencing is move rapidly into the mainstream, end users are demanding unmatched performance, reliability, stability, and security. Phone voice Call is simple connection between two parties through phone, mobile or any other voice applications over IP or other transmission media. Video (voice + Data), $\mathrm{VC}$ is allow two or more locations to start communicates video 
and audio by simultaneous two-directions. The transport protocol TCP -UPD with the unique features for each it might suite with application more than other depends on nature of the applications type and the protocol characteristic.

\section{Simulation Model}

This section presents the system model that used in our case study. Fig. 1 illustrated the layout of system which designed to support several types of modulation techniques corresponding to name of end-user workstation.

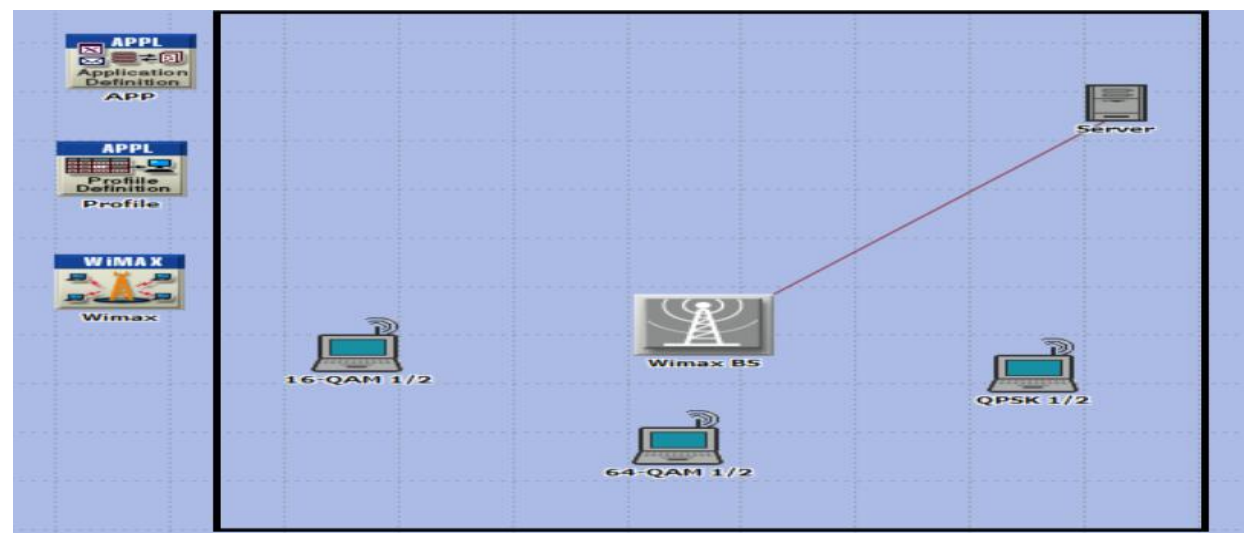

Fig. 1 Network model

Network consists of 3 users nodes. The nodes are randomly placed, each node support certain modulation with different coding rate $(1 / 2)$. The BS support adaptive modulation and connected to server that offer applications profile, the PHY layer profile used is wireless OFDM 20MHZ as bandwidth, base frequency $5 \mathrm{GHZ}$ with frame duration 5 millisecond, number of subcarrier 2048 and duplexing technique (TDD)

- Application Configuration: The configuration for application defined with low-resolution for video application (128X120 pixels,9 bits/pixel and 10 frames/sec), for voice codec G.711 PCM speech used (64 $\mathrm{Kbit} / \mathrm{s} 8 \mathrm{kHz}$ sampling frequency $\times 8$ bits per sample) [8]. Some common parameters used in OPNET Simulation, listed in Table I .

\begin{tabular}{|l|l|}
\hline Attribute & Values \\
\hline TCP parameter & Default \\
\hline Traffic characteristic Service class name & Silver \\
\hline Antenna Gain (dBi) & $15 \mathrm{dBi}$ \\
\hline Maximum Transmission Power (watts) & 0.5 \\
\hline SDU SIZE (UP +DL) & 1500 \\
\hline Buffer Size (UP+DL) & $64 \mathrm{~KB}$ \\
\hline MAC address & Auto assigned \\
\hline
\end{tabular}

Table I: Simulation Parameters

\section{Performance Metrics}

The evaluation includes some performance factors depend on:-

- A)BLER - SNR:- block error rate (BLER) defined as the ratio of the number of erroneous blocks received to the total number of blocks sent [7], Signal to noise ratio SNR, for uplink the Base station calculates SNR by received power while for downlink the subscriber station calculates and send the need of its particular suitable burst profile.

- B) Delay (sec): -Represents End to End Delay that is the time taken for a packet being transmitted across a network from source to destination depends on physical medium and associated propagation time.

- C) Jitter:- difference in the delay of the received packets, For voice over IP the receiver end when receive Real-Time Protocol (RTP) audio match the jitter and use de-jitter buffering mechanism for good QoS [6].

- D) MOS: - The MOS Mark the realized voice quality of a VoIP conversation and range from 1 (worst) to 5 (best).

\section{Simulation Result}

The performance of the WiMAX network that supports different types of modulation plus video and voice applications have been analyzed- studied and output results presented by using Discrete Event Simulator OPNET 
Performance Evaluation of Voice and video conferencing For WIMAX Network under..

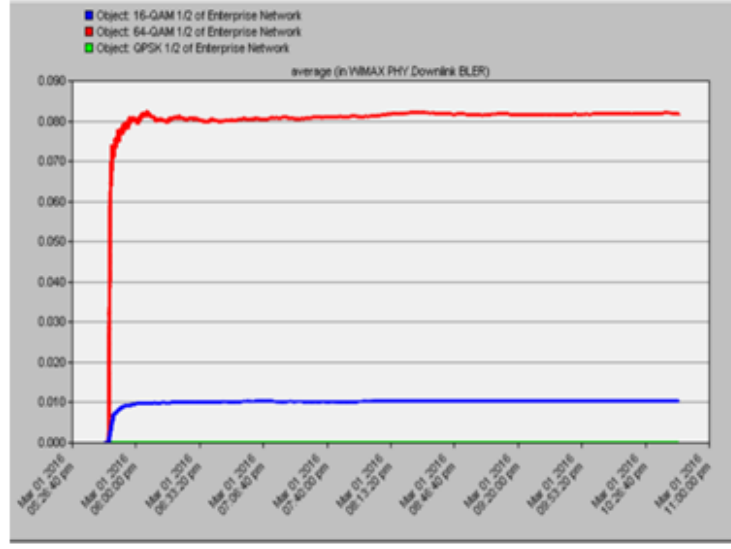

Fig. 2 Average PHY Downlink BLER

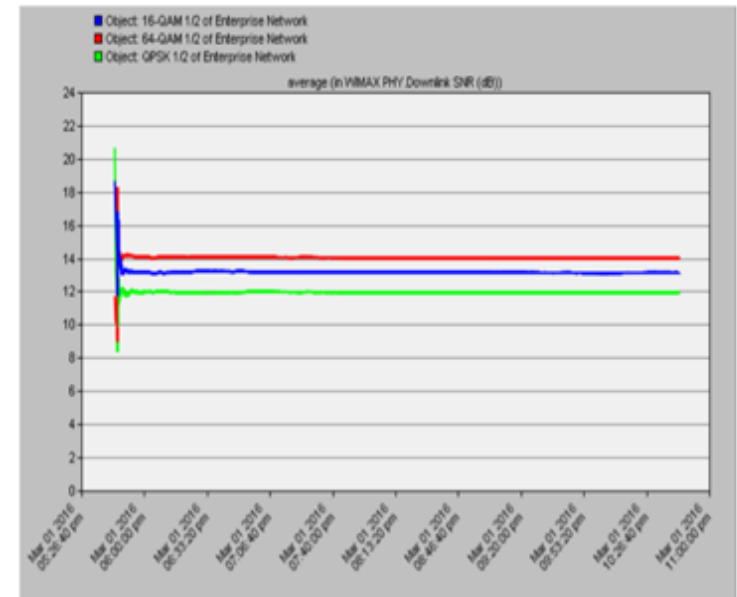

Fig.4 Average PHY Downlink SNR

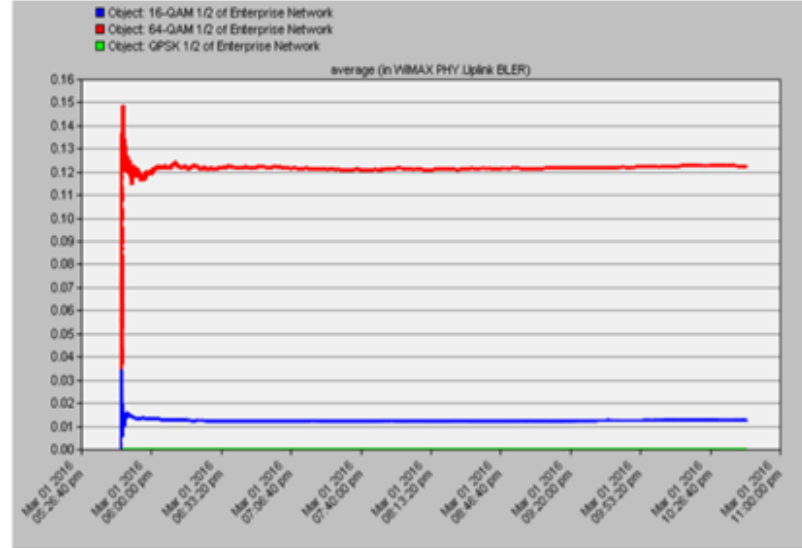

Fig. 3 Average PHY Uplink BLER

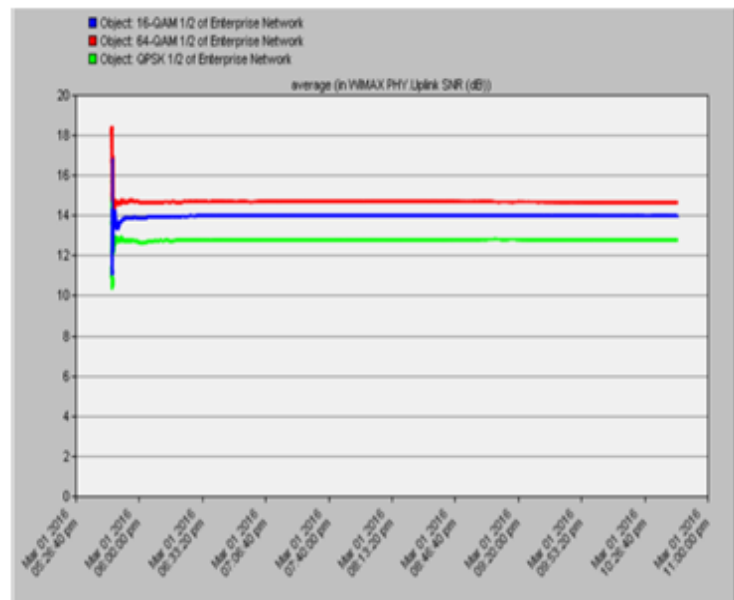

Fig.5 Average PHY Uplink SNR

A.Average PHY BLER/SNR : (Figs.2\&3) performance of QPSK $1 / 2$ has the zero reading for uplink and downlink BELR and the lowest value for SNRs that illustrated in (Figs 4\&5), 64 QAM has the highest value in both uplink/ down link BLER \& SNR as in (Figs .2\&3) and (Figs .4\&5). Observed 16 QAM 1/2 always take intermediate value between BLER / SNR values as (Figs .2\&3) \& (Figs .4\&5) present, the modulation techniques show the same order in uplink/downlink for BLER and SNR parameters.

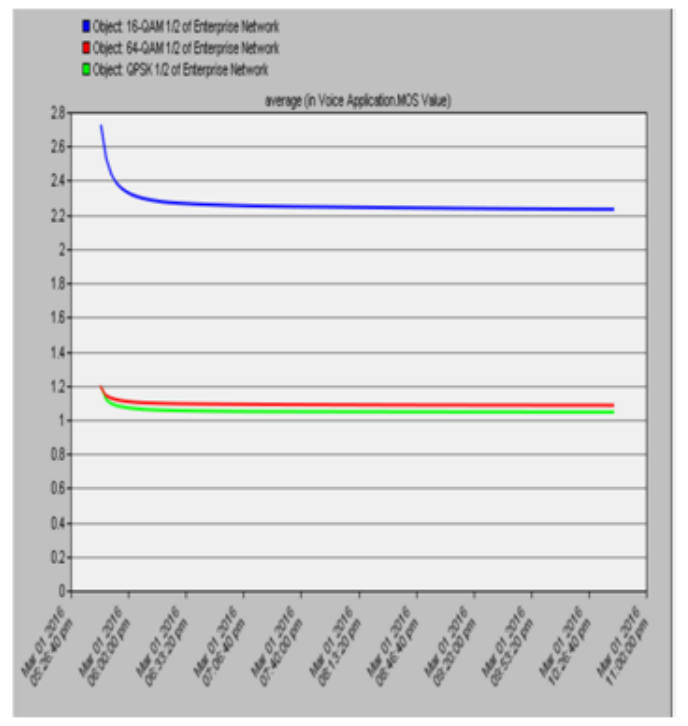

Fig. 6 Average voice application MOS Value

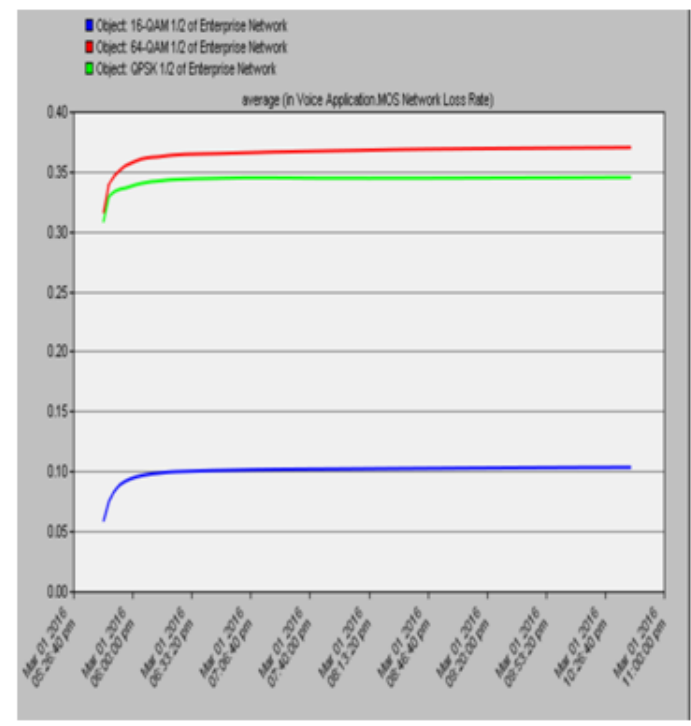

Fig. 7 Average voice application MOS Network Loss rate 
B.Average voice application MOS - MOS Network Loss rate: - MOS provides a numerical measurement of quality of voice signal transmitted, 16 QAM $1 / 2$ has the higher MOS approx. 2.2, While other techniques present similar value approx. 1.1 which consider a low quality as (Fig.6), the codec used for the voice is PCM speech. The MOS network loss illustrates performance that has the highest MOS loss rate for 64QAM $1 / 2$ although it has better value in MOS than QPSK 1/2. But QPSK $1 / 2$ in MOS loss has less reading than 64 QAM 1/2. The better performance is for 16 QAM $1 / 2$ in MOS and MOS loss rate.

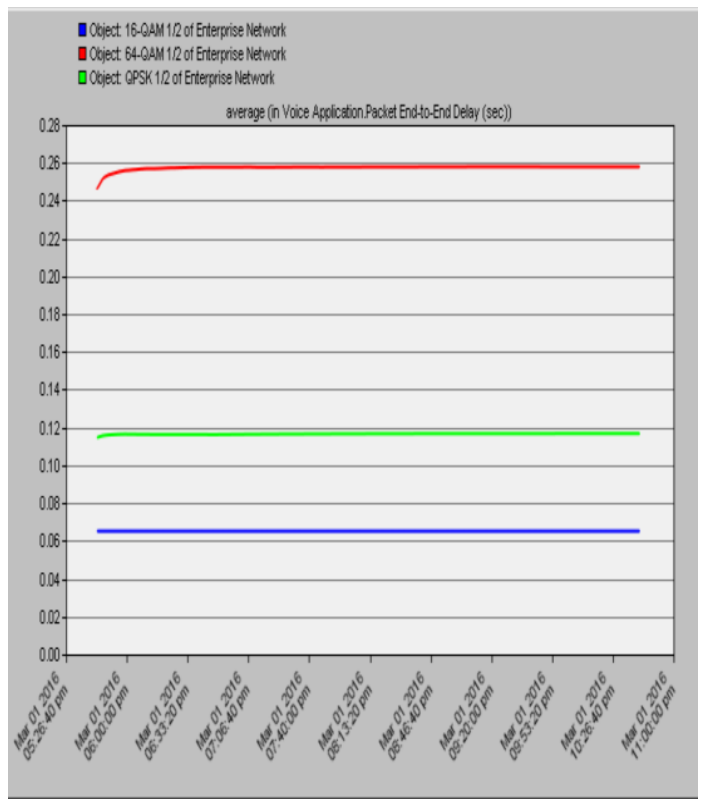

Fig. 8 Average Voice Packet End to end delay

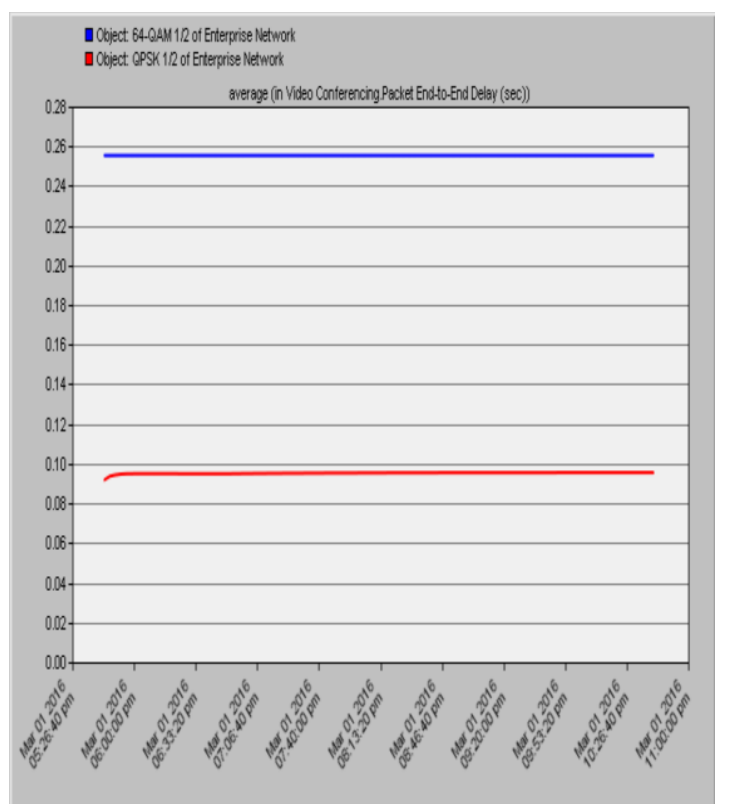

Fig. 9 Average Video Packet End to end delay

C.Average voice/video packet end to end delay:- (Fig. 8) represent the trend of voice end to end packet delay which 64 QAM 1/2 has the highest value approx. 0.26 packet end to end delay /sec. QPSK registered the in between value approx. 0.12 packet end to end delay /sec and the 16 QAM has the lowest value approx. 0.06 , While in video application (Fig.9) the scenario is different 64 QAM $1 / 2$ has the highest packet end to end delay /sec approx. 0.26, QPSK $1 / 2$ give the reading approx. 0.10 packet end to end delays $/ \mathrm{sec}$ and 16 QAM $1 / 2$ show no record.

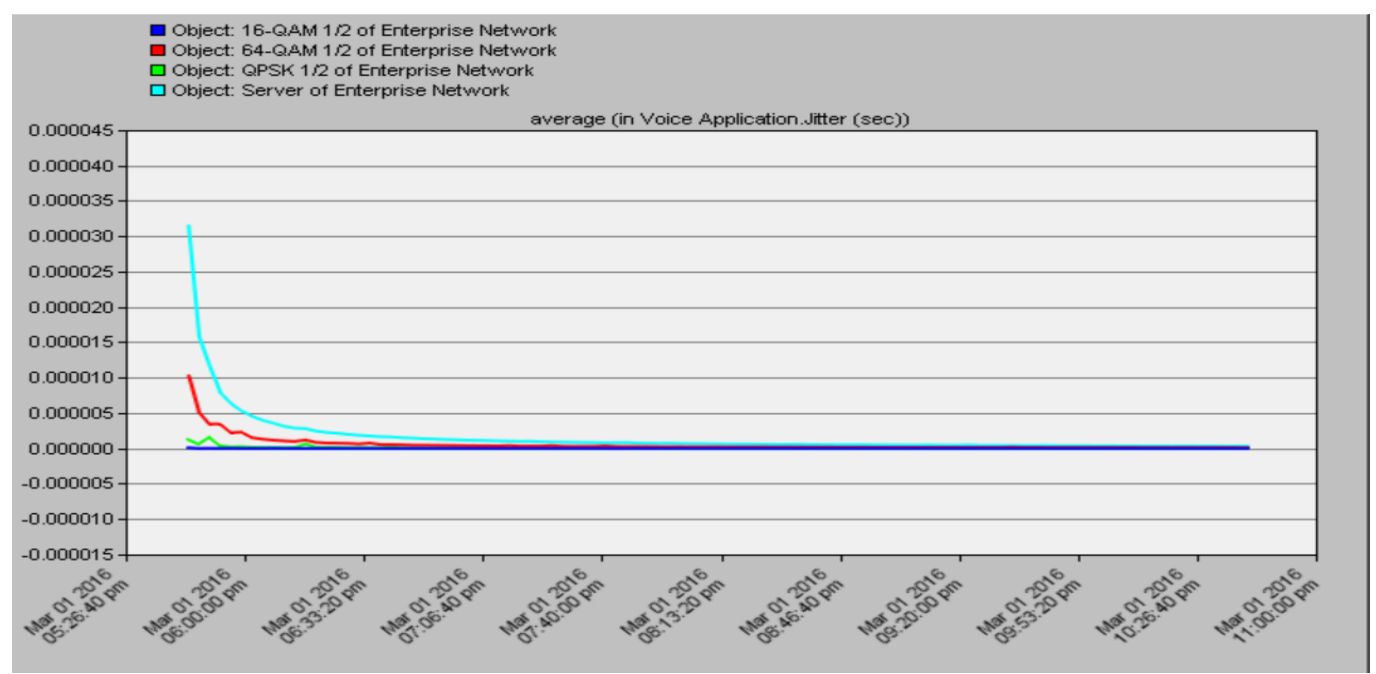

Fig. 10 Average voice application Jitter

D.Average voice Jitter: - (Fig. 10) it's summarize that the network with all modulation techniques has the same value, but the initial value it's different from technique to other. the 16-QAM 1/2 Shows unique pattern which the first value and last value is same equal to zero, same technique present lowest value in end to end delay as shown in (fig.8). For the server the value decreased after about three hours to be close to zero, server has the highest startup jitter value than other network nodes approx. $0.000030 \mathrm{sec}$. 


\section{Conclusion}

The WIMAX simulation study performed of this network, that consisting of three workstations each one support different Modulation techniques than the other and has the same voice and video application setting and connected to a server, analyzing the behavior of WIMAX network for each workstation with respect to WIMAX Physical Uplink and Downlink BLERS, SNR, voice MOS and MOS loss, voice jitter and end to end delay and the results collected by OPNET modeler using sample shows that WIMAX PHY uplink BLERs is Higher than downlink BLERs for all modulation techniques. In the SNRs Uplink for all modulation techniques used illustrated high values when compare it to Downlink SNRs values. The Voice MOS reflected a good value for 16 QAM $1 \frac{1}{2}$ since rest types have fewer values even in MOS loss rate 16 QAM $1 \frac{1}{2}$ appear again but as less loss value, Video end to end delay has the highest values when compare it with voice for all modulation techniques except the voice 64 QAM $1 / 2$ went out of this pattern. The voice application jitter has same value for all modulation techniques and the server even which zero.

\section{References}

[1] Jeffrey G. Andrews, Ph.D, Arunabha Ghosh, Ph.D, "Fundamentals of WiMAX Understanding Broadband Wireless Networking," First Edition, Prentice Hall, 2007

[2] Ramjee Prasad 1 Fernando J. Velez "WiMAX Networks Techno-Economic Vision and Challenges" Library of Congress Control Number: 2010928698

[3] A. Ghosh et al., Feb. 2005: "Broadband Wireless Access with WIMAX/802.16: Current Performance Benchmarks and Future Potential," IEEE Commune. Mag., vol. 43, pp. 129-36

[4] SYED AHSON MOHAMMAD ILYAS, WIMAX Applications ISBN 9781420045474,2008 by Taylor \& Francis Group, LLC

[5] ANDREW S. TANENBAUM Vrije Universiteit Amsterdam, The Netherlands, DAVID J. WETHERALL University of Washington Seattle, WA "computer networks fifth edition"

[6] http://www.cisco.com/c/en/us/support/docs/voice/voice-quality/18902-jitter-packet-voice.

[7] https://en.wikipedia.org/wiki/Block_Error_Rate

[8] OPNET Modeler 14.5 Documentation

[9] http://www.radio-electronics.com/

[10] IETF rfc793I. https://www.ietf.org/rfc/rfc793.txt 